\title{
NEW TOPOLOGICAL EXTENSION PROPERTIES
}

\author{
TOSHIJI TERADA
}

\begin{abstract}
A topological property $\mathscr{P}$ is called an extension property if $\mathscr{P}$ is closed-hereditary and productive. In this paper new topological extension properties are introduced, which to some extent fill up a gap between complete regularity and compactness.
\end{abstract}

All spaces considered here are Tychonoff. As generalizations of compactness, major topological properties have been investigated (e.g. realcompactness, topological completeness, etc.). Mostly these properties are closed-hereditary and productive. A topological property $\mathcal{P}$ is called an extension property if $\mathcal{P}$ is closed-hereditary and productive. Some details and numerous examples of extension properties can be found in [6].

In this paper, as generalizations of compactness and first countability, we introduce new topological extension properties which fill a gap between complete regularity and compactness.

We review some notation: For a cardinal $m$ the cardinal successor of $m$ is denoted by $\mathrm{m}^{+}$. For an ordinal $\alpha$ the ordinal successor of $\alpha$ is denoted by $\alpha+1$. We regard each cardinal as its initial ordinal. For any ordinal $\gamma, W(\gamma)$ denotes the space of all ordinals less than $\gamma$ with the usual interval topology.

1. $P_{z}(\mathrm{~m})$-compactness and $P_{z}$-compactness. A covering $\mathscr{Q}$ of a space $X$ is called a partition if each pair of members in $Q_{L}$ is disjoint. Throughout this paper each member of a partition is assumed to be nonempty. A partition $\mathcal{U}$ is called a $z$-partition if each member of $\mathscr{U}$ is a zero-set. For a space $X$ the Stone-Čech compactification of $X$ is denoted by $\beta X$.

Definition 1. Let $m$ be an infinite cardinal. A space $X$ is called $P_{z}(\mathrm{~m})$ compact if for any $y \in \beta X-X$ there is a $z$-partition $\mathcal{Q}$ of $X$ such that $|Q|<\mathfrak{m}$ and such that $y \notin \mathrm{cl}_{\beta X} Z$ for any $Z \in \mathcal{U}$. A space $X$ is called $P_{z}$-compact if $X$ is $P_{z}(\mathrm{~m})$-compact for some cardinal $\mathrm{m}$.

Obviously for infinite cardinals $\mathfrak{m}$ and $\mathfrak{n}$ such that $\mathfrak{m} \leqslant \mathfrak{n}, P_{z}(\mathfrak{m})$-compactness implies $P_{z}(\mathfrak{n})$-compactness. It is also obvious that a space $X$ is $P_{z^{-}}$ compact if and only if for any $y \in \beta X-X$ there is a $z$-partition $\mathcal{Q}$ of $X$ such that $y \notin \mathrm{cl}_{\beta X} Z$ for any $Z \in \mathcal{Q}$.

We will omit the proof of the following simple lemma.

Received by the editors November 8, 1976 and, in revised form, April 19, 1977.

AMS (MOS) subject classifications (1970). Primary 54D30; Secondary 54G10.

Key words and phrases. Extension property, zero-set, partition, Stone-Čech compactification, realcompactness, $P$-space. 
LEMMA 1. For a space $X$ the following are equivalent.

(1) $X$ is $P_{z}(\mathfrak{m})$-compact.

(2) There exists a compactification $\mathrm{c} X$ of $X$ with the property that for any $y \in \mathrm{c} X-X$ there is a $z$-partition $\mathscr{Q}$ of $X$ such that $|\mathcal{Q}|<\mathrm{m}$ and such that $y \notin \mathrm{cl}_{\mathrm{c} X} Z$ for any $Z \in \mathcal{Q}$.

THEOREM 1. (1) For any infinite cardinal $m, P_{z}(m)$-compactness is an extension property.

(2) $P_{z}$-compactness is an extension property.

Proof. (1) Let $Y$ be a $P_{z}(\mathfrak{m})$-compact space and let $X$ be a closed subspace of $Y$. Take $\operatorname{cl}_{\beta Y} X$ as a compactification of $X$. Then obviously condition (2) of Lemma 1 is satisfied. Hence $X$ is $P_{z}(\mathrm{~m})$-compact. For a product $X$ of $P_{z}(\mathfrak{m})$-compact spaces $\left\{Y_{\lambda}: \lambda \in \Lambda\right\}$, if we take the compactification $\mathrm{c} X=$ $\Pi\left\{\beta Y_{\lambda}: \lambda \in \Lambda\right\}$ of $X$, then condition (2) of Lemma 1 is satisfied. Hence $X$ is $P_{z}(\mathfrak{m})$-compact.

(2) $P_{z}$-compactness is obviously closed-hereditary. Now let $\left\{Y_{\lambda}: \lambda \in \Lambda\right\}$ be a set of $P_{z}$-compact spaces. Then for each $\lambda \in \Lambda$ there is a cardinal $m_{\lambda}$ such that $Y_{\lambda}$ is $P_{z}\left(m_{\lambda}\right)$-compact. Since $\Lambda$ is a set, there is a cardinal $m$ such that $\mathfrak{m} \geqslant \mathfrak{m}_{\lambda}$ for each $\lambda \in \Lambda$. Then each $Y_{\lambda}$ is $P_{z}(\mathrm{~m})$-compact by the remark after Definition 1, so $\Pi\left\{Y_{\lambda}: \lambda \in \Lambda\right\}$ is $P_{z}(\mathrm{~m})$-compact by (1). Hence $P_{z}$-compactness is productive.

THEOREM 2. The following are equivalent for a space $X$.

(1) $X$ is compact.

(2) $X$ is $P_{z}\left(\aleph_{0}\right)$-compact.

Proof. Compact spaces are obviously $P_{z}\left(\aleph_{0}\right)$-compact. The converse is also obvious since $\cup\left\{\mathrm{cl}_{\beta X} Z: Z \in \mathcal{Q}\right\}=\beta X$ for any finite $z$-partition $\mathscr{Q}$ of $X$.

Next, we study a relation between $P_{z}(\mathfrak{m})$-compactness and realcompactness.

THEOREM 3. (1) $P_{z}\left(\aleph_{1}\right)$-compact spaces are realcompact.

(2) Realcompact spaces are $P_{z}\left(\left(2^{\aleph_{0}}\right)^{+}\right)$-compact.

Proof. (1) Let $X$ be a $P_{z}\left(\aleph_{1}\right)$-compact space. Then for any $y \in \beta X-X$ there is a countable $z$-partition $\mathcal{Q}$ of $X$ such that $y \notin \mathrm{cl}_{\beta X} Z$ for any $Z \in \mathcal{Q}$. Hence there is a zero-set $Z_{y}$ of $\beta X$ such that $y \in Z_{y}$ and $Z_{y} \cap X=\varnothing$. So $X$ is realcompact.

(2) Let $X$ be a realcompact space. Then for any $y \in \beta X-X$ there is a real-valued continuous function $f$ on $\beta X$ such that $f(y)=0$ and $f(x)>0$ for any $x \in X$. Now let $\mathscr{Q}_{y}=\left\{f^{-1}(r) \cap X: r \in \mathbf{R}, f^{-1}(r) \cap X \neq \varnothing\right\}$. Then obviously $\mathscr{Q}_{y}$ is a $z$-partition of $X$ such that $\left|\mathcal{Q}_{y}\right|<\left(2^{\kappa_{0}}\right)^{+}$, and $y \notin \mathrm{cl}_{\beta X} Z$ for any $Z \in \mathscr{Q}_{y}$.

EXAMPLE 1. The converse of (1) in Theorem 3 is not true. In fact, since the real line $\mathbf{R}$ with the usual interval topology cannot have an infinite countable $z$-partition [4, p. 195], $\mathbf{R}$ is not $P_{z}\left(\boldsymbol{\aleph}_{1}\right)$-compact.

ExAmple 2. The converse of (2) in Theorem 3 is not true. Since each point 
of $W\left(\omega_{1}\right)$ is a zero-set, where $\omega_{1}$ is the first uncountable ordinal, $W\left(\omega_{1}\right)$ is $P_{z}\left(\aleph_{2}\right)$-compact and hence $P_{z}\left(\left(2^{\aleph_{0}}\right)^{+}\right)$-compact. But $W\left(\omega_{1}\right)$ is not realcompact.

The referee requests a result that for any uncountable cardinal $m$ there is a space $X$ which is $P_{z}(\mathrm{~m})$-compact, but not $P_{z}(\mathrm{n})$-compact for any $\mathrm{n}<\mathrm{m}$. The following partially answers this request.

THEOREM 4. Let $m$ be an uncountable cardinal. If

(a) $\mathfrak{m}=\mathfrak{f}^{+}$where $\mathfrak{f}^{\prime}$ is a regular cardinal, or

(b) $\mathrm{m}$ is a limit cardinal,

then there is a $P_{z}(\mathfrak{m})$-compact space which is not $P_{z}(\mathfrak{n})$-compact for any $\mathfrak{n}<\mathfrak{m}$.

Proof. (a) It is obvious that $W\left(\omega_{0}\right)$ is a $P_{z}\left(\kappa_{1}\right)$-compact space which is not $P_{z}\left(\aleph_{0}\right)$-compact, where $\omega_{0}$ is the first infinite ordinal. Now let be an uncountable regular cardinal, and let

$$
V\left(\mathfrak{f}^{+}\right)=W(\mathfrak{f})-\left\{\alpha \in W(\mathfrak{f}): \operatorname{cf}(\alpha)>\omega_{0}, \alpha \text { is a limit ordinal }\right\},
$$

where $\operatorname{cf}(\alpha)$ is the cofinality of $\alpha$. Then $V\left(\mathfrak{f}^{+}\right)$is $P_{z}\left(\mathfrak{f}^{+}\right)$-compact since each point of $V\left(\mathfrak{f}^{+}\right)$is a zero-set and $\left|V\left(\mathfrak{f}^{+}\right)\right|<\mathfrak{f}^{+}$. On the other hand, it is obvious that $\beta V\left(\mathfrak{f}^{+}\right)=W(\mathfrak{f}+1)$. By the regularity of $\mathfrak{f}$, for any $z$-partition $\mathcal{Q}$ of $V\left(\mathfrak{f}^{+}\right)$such that $|\mathscr{U}|<\mathfrak{f}$, there is a member of $Z$ of $\mathscr{U}$ which is cofinal in $V\left(\mathfrak{f}^{+}\right)$. This shows that the point $\mathfrak{f}^{\mathfrak{a}}$ of $\beta V\left(\mathfrak{f}^{+}\right)-V\left(\mathfrak{f}^{+}\right)$is contained in $\cup\left\{\operatorname{cl}_{\beta V\left(\mathfrak{t}^{+}\right)} Z: Z \in \mathscr{Q}\right\}$ for any $z$-partition $\mathscr{U}$ of $V\left(\mathfrak{f}^{+}\right)$such that $|\mathcal{Q}|<\mathfrak{t}$. That is, $V\left(\mathfrak{f}^{+}\right)$is not $P_{z}(\mathfrak{f})$-compact and hence not $P_{z}(\mathfrak{n})$-compact for any $\mathfrak{n}<\mathfrak{i}^{+}$.

(b) Let $m$ be a limit cardinal. Then there is a transfinite sequence $\left\{f_{\lambda}: \lambda \in\right.$ $\Lambda$ \} of regular cardinals such that $\mathfrak{f}_{\lambda}<\mathfrak{m}$ for any $\lambda \in \Lambda$ and $\sup \left\{\mathfrak{f}_{\lambda}: \lambda \in \Lambda\right\}$ $=\mathfrak{m}$. Now for each $\lambda \in \Lambda$ let $V\left(\mathfrak{H}_{\lambda}^{+}\right)$be the space constructed in the proof of (a). Then the product space $\Pi\left\{V\left(\mathfrak{f}_{\lambda}^{+}\right): \lambda \in \Lambda\right\}$ is obviously $P_{z}(\mathfrak{m})$-compact, but not $P_{z}(\mathfrak{n})$-compact for any $\mathrm{n}<\mathrm{m}$.

Let $E$ be a space. Then a space $X$ is called $E$-compact if $X$ is homeomorphic to a closed subspace of the topological power $E^{\mathfrak{m}}$ for some cardinal $\mathrm{m}[\mathbf{1}]$.

Corollary. There does not exist a space $E$ such that $P_{z}$-compactness is equivalent to E-compactness.

Proof. By Theorem 4, for any $P_{z}$-compact space $E$ there is a $P_{z}$-compact space $X$ which cannot be homeomorphic to a closed subspace of the topological power $E^{\mathfrak{m}}$ for any cardinal $\mathrm{m}$.

THEOREM 5. (1) Let $X$ be a closed subspace of a product of spaces of which each point is $a G_{\delta}$-set. Then $X$ is $P_{z}$-compact.

(2) Topologically complete spaces are $P_{z}$-compact.

Proof. (1) Since spaces of which each point is a $G_{\delta}$-set are obviously $P_{z}$-compact, this statement follows from the fact that $P_{z}$-compactness is an extension property.

(2) Since topologically complete spaces are closed subspaces of products of 
metrizable spaces, (2) is a special case of (1).

It seems natural to ask whether every space is $P_{z}$-compact. In the next section we will show the existence of a space which is not $P_{z}$-compact.

2. $P$-realcompactness. A point $x$ of a space $X$ is a $P$-point if any $G_{\delta}$-set containing $x$ is a neighborhood of $x$. A space $X$ is a $P$-space if every point of $X$ is a $P$-point. For a space $X, \mathrm{p} X$ denotes the space with the underlying set equal to the underlying set of $X$ and with the topology generated by all $G_{\delta}$-sets of $X$ (see [3]).

Definition 2. A space $X$ is called $P$-realcompact if $\mathrm{p} X$ is realcompact.

LemMa 2 (CF. R. E. WheELER [5]). Realcompact spaces are P-realcompact.

The proof of Lemma 2 is essentially the same as that of Wheeler [5] which shows that if $X$ is topologically complete, so is $\mathrm{p} X$. The following lemma is trivial.

Lemma 3. Let $\left\{X_{\lambda}: \lambda \in \Lambda\right\}$ be a set of spaces. Then $\operatorname{pII}\left\{X_{\lambda}: \lambda \in \Lambda\right\}=$ $\mathrm{p} \Pi\left\{\mathrm{p} X_{\lambda}: \lambda \in \Lambda\right\}$.

THEOREM 6. P-realcompactness is an extension property.

Proof. Let $X$ be a closed subspace of a $P$-realcompact space $Y$. Then since for any $G_{\delta}$-set $G$ of $X$ there is a $G_{\delta}$-set $G^{\prime}$ of $Y$ such that $G=G^{\prime} \cap X, \mathrm{p} X$ is a closed subspace of $\mathrm{p} Y$. Hence $\mathrm{p} X$ is realcompact since $\mathrm{p} Y$ is realcompact. Next, let $\left\{X_{\lambda}: \lambda \in \Lambda\right\}$ be a set of $P$-realcompact spaces. Then $\Pi\left\{\mathrm{p} X_{\lambda}: \lambda \in\right.$ $\Lambda\}$ is realcompact as a product of realcompact spaces. By Lemma 2, $\mathrm{p} \Pi\left\{\mathrm{p} X_{\lambda}: \lambda \in \Lambda\right\}$ is realcompact. Hence $\mathrm{p} \Pi\left\{X_{\lambda}: \lambda \in \Lambda\right\}$ is realcompact by Lemma 3.

Theorem 7. Let $X$ be a nonmeasurable $P_{z}$-compact space. Then $X$ is P-realcompact.

Proof. We can assume that $X$ is not realcompact by Lemma 2. By the definition of $P_{z}$-compactness, for any $y \in v X-X$ there is a $z$-partition $\mathscr{U}_{y}$ of $X$ such that $\mathrm{cl}_{v X} Z \not \nexists y$ for any $Z \in \mathcal{Q}_{y}$ where $v X$ is the Hewitt realcompactification of $X$. Then since $\mathrm{cl}_{v X} Z$ is a zero-set of $v X$ for any zero-set $Z$ of $X$, $\left\{\mathrm{cl}_{v X} Z: Z \in \mathscr{Q}_{y}\right\}$ is a $z$-partition of $\cup\left\{\mathrm{cl}_{v X} Z: Z \in \mathscr{Q l}_{y}\right\}$. Further $\mathrm{cl}_{v X} Z$ is realcompact for any $Z \in \mathcal{Q}_{y}$. Hence $\operatorname{pcl}_{v X} Z$ is realcompact for any $Z \in \mathcal{Q l}_{y}$ by Lemma 2. Then $\mathrm{p} \cup\left\{\mathrm{cl}_{v X} Z: Z \in \mathscr{Q}_{y}\right\}$ is a topological sum of realcompact spaces $\left\{\operatorname{pcl}_{v X} Z: Z \in \mathcal{Q}_{y}\right\}$ since each set of the form $\mathrm{cl}_{v X} Z$, with $Z$ a zero-set of $X$, is a zero-set of $v X$. Now $\left|\mathcal{Q}_{y}\right|$ is nonmeasurable, hence $\mathrm{p} \cup\left\{\operatorname{cl}_{v X} Z: Z \in\right.$ $\left.\mathcal{Q}_{y}\right\}$ is realcompact. Since

$$
\begin{aligned}
X & =\cap\left\{\cup\left\{\mathrm{cl}_{v X} Z: Z \in \mathcal{Q}_{y}\right\}: y \in v X-X\right\}, \\
\mathrm{p} X & =\cap\left\{\mathrm{p} \cup\left\{\mathrm{cl}_{v X} Z: Z \in \mathcal{Q}_{y}\right\}: y \in v X-X\right\} .
\end{aligned}
$$

Then $\mathrm{p} X$ is realcompact as an intersection of realcompact spaces.

COROLlaRY. The following are equivalent. 
(1) There is a $P_{z}$-compact space which is not $P$-realcompact.

(2) There is a measurable cardinal.

Proof. If there is a measurable cardinal, then there is a discrete space $D$ which is not realcompact. $D$ is a $P_{z}$-compact space which is not $P$-realcompact.

Example 3. There is a nonmeasurable space which is not $P$-realcompact. Hence there is a space which is not $P_{z}$-compact by Theorem 7. In fact there is a nonmeasurable $P$-space which is not realcompact (see $9 \mathrm{~L}$ of [2]).

REMARK. If $\mathrm{p} X$ is Lindelöf or discrete, then $X$ is $P_{z}$-compact. However the author does not know whether or not there exists a $P$-realcompact space which is not $P_{z}$-compact.

The author wishes to express his thanks to the referee for his helpful suggestions.

\section{REFERENCES}

1. R. Engelking and S. Mrówka, On E-compact spaces, Bull. Acad. Polon. Sci. Sér. Sci. Math. Astronom. Phys. 6 (1958), 429-435.

2. L. Gillman and M. Jerison, Rings of continuous functions, Van Nostrand, Princeton, N.J., 1960.

3. A. Misra, A topological view of P-spaces, General Topology and Appl. 2 (1972), 349-362.

4. L. A. Steen and J. A. Seebach, Jr., Counterexamples in topology, Holt, New York, 1970.

5. R. E. Wheeler, On separable z-filters, General Topology and Appl. 5 (1975), 333-345.

6. R. G. Woods, Topological extension properties, Trans. Amer. Math. Soc. 210 (1975), 365-385.

Department of Mathematics, University of Tsukuba, Sakuramura Ibaraki, 300-31, JAPAN 